\title{
Rede de colaboração científica sobre oleaginosas envolvidas na produção do biodiesel
}

Isadora Lucena Andrade

Mestre em Bioenergia pela Faculdade de Tecnologia e Ciências (FTC). Professora do Centro de Educação Profissional e Especialização.

Cláudio Marcelo Matos Guimarães

Mestre em Bioenergia pela Faculdade de Tecnologia e Ciências (FTC). Professor Mestre da Faculdade SENAI CIMATEC.

Viviane Galvão

Doutora em Biotecnologia pela Universidade Estadual de Feira de Santana (UEFS). Professora Doutora do Programa de Pós-Graduação em Bioenergia da Faculdade de Tecnologia e Ciências (FTC).

http://dx.doi.org/10.1590/1981-5344/2605

Redes de colaboração científica representam estratégias para compartilhamento de informações e novos conhecimentos sobre as comunidades acadêmicas. Este tipo de rede pode identificar os agentes que compõem a rede e a intensidade da ligação que une os atores. Por esta razão, neste trabalho foram identificadas as relações entre os autores, países e instituições em publicações sobre seis oleaginosas pertencentes à cadeia produtiva do biodiesel. As oleaginosas selecionados foram soja, pinhãomanso, dendê, canola, girassol e mamona. Nesta rede, os vértices são autores, países ou instituições e as arestas são obtidas por meio da análise publicação. Portanto, se dois cientistas são coautores em uma publicação, eles estão conectados. As publicações sobre estas seis oleaginosas foram recuperadas da base de dados do Web of Knowledge de 1945 a 2011. As consultas foram feitas no modo de Pesquisa Avançada, procurando o nome da oleaginosa no título da publicação e a palavra biodiesel no título, resumo ou palavras-chave. Para cada oleaginosa, três redes foram construídas: autores, países e 
instituições. No período estudado, foram analisados um total de 1378 publicações. Quatro países (Brasil, Índia, China e Estados Unidos da América) participaram das publicações sobre todas as oleaginosas estudadas.

Palavras-chaves: Análise de Redes Sociais; Coautoria; Biodiesel.

\section{Scientific collaboration network on oilseed involved in biodiesel production}

Scientific collaboration networks represent strategies for sharing information and new knowledge about academic communities. This type of network can identify the actors that compose the network and the intensity of the connection joining the actors. For this reason, in this work we identified the relationships among authors, countries and institutions in publications about six oilseeds belonging to the biodiesel production chain. The oilseeds selected were soybean, jatropha, palm, canola, sunflower, and castor. In this network, the vertices are authors, countries or institutions and the edges are obtained by the publication analysis. Therefore, if two scientists have coauthored a publication, they are connected. Additionally, the countries and institutions network are built from the authors addresses. Publications about six oilseeds were recover from the Web of Knowledge database from 1945 to 2011. Queries were made in Advanced Search mode, looking for the oilseed name in the publication title and the biodiesel word in the publication title, abstract or keywords. For each oilseed, three networks were constructed: authors, countries, and institutions. In period studied, a total of 1378 publications were analyzed. Four countries (Brazil, India, China, and United States of America) had participated in the publications about all oilseeds studied.

Keywords: Social Network Analysis; Coauthorship; Biodiesel.

\section{Introdução}


O termo bioenergia é bastante amplo e se refere à energia renovável produzida a partir de fontes biológicas. Este tema ganhou relevância nos últimos anos, mediante a constatação do aquecimento global e previsão de esgotamento de fontes energéticas provenientes do petróleo, do carvão e do gás natural (FERRARI; OLIVEIRA; SCABIO, 2005). O biocombustível apresenta uma alternativa governamental para auxiliar na redução da emissão de gases poluentes na atmosfera terrestre. Assim, muitos países estão investindo em tecnologia e mão-de-obra especializada para ampliar a produção de diversos tipos de biocombustíveis (HOLANDA, 2004).

O aumento da demanda por biocombustível está ocorrendo devido à elevação contínua do preço do petróleo e o encarecimento das matrizes energéticas de diversas nações (TÁVORA, 2011). Atualmente, este tipo de combustível tem grande importância mundial, pois ele é facilmente disponível a partir de fontes de biomassa, fornece novas oportunidades socioeconômicas no setor rural, possui um impacto ambiental reduzido, possui um considerável potencial ecológico, é biodegradável, reduz o uso de combustíveis fósseis e contribui para a sustentabilidade (PUPPAN, 2002). Os biocombustíveis mais comumente utilizados comercialmente são o etanol, biodiesel e biogás.

O biodiesel é composto de uma mistura de alquilésteres de ácidos graxos de cadeia linear longa produzido a partir de biomassa renovável (LOBO; FERREIRA; CRUZ, 2009). O biodiesel pode ser utilizado como substituto ou adicionado ao óleo diesel derivado do petróleo (POUSA; SANTOS; SUAREZ, 2007). Este biocombustível pode utilizar como matéria-prima espécies oleaginosas ou gorduras animais. As oleaginosas comumente empregadas na produção de biodiesel são soja, girassol, canola, mamona, dendê e pinhão-manso (SILVA; GALVÃO, 2014). O biodiesel precisa atender a rigorosas especificações técnicas para poder ser comercializado.

No Brasil, as especificações do biodiesel são estabelecidas pela Agência Nacional do Petróleo, Gás Natural e Biocombustíveis, através da Resolução no 7 de 2008 (ANP, 2008). Os parâmetros estabelecidos nesta resolução fornecem resultados bastante esclarecedores quanto à qualidade do biodiesel, dentre eles pode-se destacar: massa específica, viscosidade cinemática, resíduo de carbono, teor de éster, enxofre total, índice de iodo, fração de destilados, número de cetano, ponto de fulgor, cinzas sulfatadas, ponto de névoa, ponto de entupimento de filtro a frio e ponto de fluidez (LOBO et al., 2009).

O biodiesel é obtido através de processos químicos, sendo que o mais utilizado industrialmente é a transesterificação de óleos e gorduras (GONÇALVES et al., 2014). Na transesterificação ocorre uma reação química no qual um ácido graxo (óleo vegetal) reage com um álcool (etanol ou metanol) na presença de catalisadores ácidos, básicos ou enzimáticos, formando dois compostos principais: biodiesel e glicerol. Os catalisadores alcalinos são os mais empregados, pois são mais eficientes, promovendo altos rendimentos (DABDOU; BRONZEL; RAMPIN, 2009). O glicerol ou glicerina é uma molecular polar que pode ser utilizada na 
produção de cosméticos, fármacos e na alimentação animal (BEATRIZ; ARAÚJO; LIMA, 2011). Outro método utilizado para se obter biodiesel é a esterificação. A esterificação consiste na reação de um ácido graxo com um álcool na presença de um catalisador ácido, formando um éster e uma molécula de água. As principais vantagens deste processo são produzir biodiesel a partir de resíduos de baixo valor agregado e possuir apenas água como subproduto (PERLINGEIRO, 2014).

$\mathrm{Na}$ atualidade, o interesse e o volume de pesquisas produzidas sobre o biodiesel tem aumentado significantemente. Este acontecimento acarreta na ampliação das colaborações científicas sobre este tema. Este fenômeno social estimula o pesquisador a interagir dentro de uma rede na comunidade científica (LETA; CHAIMOVICH, 2002). As redes de colaboração permitem a existência de uma nova dinâmica de relações entre pesquisadores, instituições e países que realizam publicações em conjunto, visto que um trabalho acadêmico representa uma das maneiras mais claras de se registrar as relações de colaboração no meio científico (ABBASI; ALTMANN; HWANG, 2010). O mapeamento desses atores e as relações de vínculo que os une, permite o reconhecimento dos agrupamentos formados. As conexões existentes nessas relações representam o elemento essencial de uma rede, pois refletem a natureza do elo entre os interlocutores (JOHNSON, 2011).

As interações estabelecidas entre as colaborações dos atores podem ser representadas através de redes sociais (WASSERMAN; FAUST, 2007). A Análise de Redes Sociais (ARS) consiste no estudo de uma estrutura formada por um conjunto finito de atores com algum grau de relacionamento. No caso de colaboração científica, um ator pode ser um pesquisador, instituição ou país (GLANZEL, 2002). A utilização da ARS pode levar a identificação dos grupos que estão ativamente pesquisando em uma determinada área de conhecimento e a influência dos diferentes grupos dentro da comunidade pesquisada (NEWMAN, 2001). Esta metodologia vem sendo utilizada com êxito na academia, pois investiga como os trabalhos em cooperação estão sendo publicados. Assim, diversos autores têm realizado trabalhos a respeito de ARS (BARBASTEFANO et al., 2013; NEWMAN, 2001; GOLDENBERG et al., 2010).

Uma grande quantidade de trabalhos sobre colaboração científica já foram desenvolvidos em diversas áreas do conhecimento, como por exemplo: padrão de colaboração acadêmica em trabalhos sobre biodiesel (ANDRADE; GALVÃO, 2014) e biogás (GUIMARÃES; GALVÃO, 2014; GUIMARÃES; GALVÃO, 2015); análise das publicações na base de dados da American Physical Society (BRAGA; PEREIRA; MORET, 2014), na revista científica Química Nova (SOUZA; BARBASTEFANO; LIMA, 2012) e no periódico Biomass and Bioenergy (DE SOUZA; GALVÃO, 2014); perfil de trabalhos relacionados ao biodiesel (SALES; AMORIM; PINTO, 2006), educação física (REVERTER-MASÍA et al., 2014), educação especial (HAYASHI; HAYASHI; LIMA, 2008) e sociologia (MARCELO; HAYASHI, 2013); mapeamento das publicações sobre mineração de textos biomédicos (WOSZEZENKI; GONÇALVES, 2013); produção de trabalhos 
apresentados nos principais eventos brasileiros na área de Ciência da Informação (ARBOIT; BUFREM, 2011); colaboração entre programas de pós-graduação brasileiros em ciência da informação (SILVA et al., 2012); produção científica na área de ciências biológicas pela Universidade Federal do Rio Grande do Sul (SANTIN; VANZ; STUMPF, 2015) e análise bibliométrica de trabalhos sobre doenças negligenciadas (MOREL et al., 2009), doença de chagas (GONZÁLEZ-ALCAIDE et al., 2012), tuberculose (VASCONCELOS; MOREL, 2012) e leishmaniose (RAMOS; GONZÁLEZALCAIDE; BOLAÑOS-PIZARRO, 2013).

O fenômeno social emergindo a partir dos trabalhos acadêmicos publicados em parceria está sendo bastante pesquisado na contemporaneidade. Assim, a justificativa desse artigo está embasada no crescimento da quantidade de trabalhos publicados em colaboração com a temática de bioenergia. Esse acréscimo acarreta na necessidade de verificar como esse tipo de cooperação está se comportando. Desta forma, o presente artigo tem como objetivo analisar o perfil da rede de colaboração científica entre pesquisadores que publicaram sobre as principais oleaginosas envolvidas na produção do biodiesel a partir das relações de coautoria presentes nos trabalhos selecionados. As seis oleaginosas escolhidas foram: soja, pinhão-manso, dendê, canola, girassol e mamona. Estas relações de colaboração foram obtidas a partir de documentos indexados na base de dados Web of Science do Institute for Scientific Information Web of Knowledge, entre o período de 1945 a 2011.

\section{Colaboração científica e coautoria}

A colaboração científica pode ser definida como o trabalho desenvolvido por dois ou mais pesquisadores trabalhando conjuntamente para atingir um objetivo comum e, assim, produzir novos conhecimentos. Neste tipo de cooperação pode haver compartilhamento de recursos intelectuais, econômicos e/ou físicos (KARTZ; MARTIN, 1997). Entretanto, a contribuição de cada pesquisador pode se dar em níveis diferentes, desde a simples expressão de uma opinião sobre a pesquisa até o trabalho conjunto durante todo o projeto (BORDONS; GOMEZ, 2000). O trabalho compartilhado é estimulado pelas agências de fomento a pesquisa, pois normalmente proporciona economia de tempo, recursos financeiros e/ou materiais (MAIA; CAREGNATO, 2008). Adicionalmente, a junção de competência e a união de esforços entre os pesquisadores impulsionam a produção de conhecimento (BALANCIERI et al., 2005). A colaboração científica pode ocorrer no domínio dos colégios invisíveis, ou seja, grupos informais de indivíduos que compartilham os mesmos interesses acadêmicos (OTTE; ROUSSEAU, 2002).

O termo colaboração científica muitas vezes é considerado sinônimo de coautoria. Contudo, o estudo da coautoria não mede a colaboração em todo o seu contexto e complexidade, devido ao fato de que nem sempre todos os autores de uma publicação acadêmica são responsáveis pelo trabalho intelectual (KARTZ; MARTIN, 1997; VANZ; STUMPF, 2010). De forma oposta, uma colaboração científica também pode não resultar em 
coautoria, visto que os colaboradores podem publicar isoladamente os resultados em periódicos específicos de sua área (BORDONS; GÓMEZ, 2000). Assim, a coautoria de uma publicação evidencia uma relação oficial de dois ou mais autores ou organizações (LAUDEL, 2002). Atualmente, a quantidade de trabalhos em colaboração científica está aumentando e a coautoria tem sido largamente utilizada por muitos pesquisadores para investigar como está ocorrendo o intercâmbio acadêmico (KARTZ; MARTIN, 1997).

O impacto e a visibilidade de uma publicação está fortemente correlacionado com o número de autores, instituições e países presentes, pois quanto maior essa quantidade maior é o alcance do artigo. Assim, o aumento na possibilidade de citações cresce com a coautoria (GLANZEL, 2002; PERSSON; GLÄNZEL; DANELL, 2004). Adicionalmente, a produtividade (em termos de publicação) de um pesquisador está correlacionada com os níveis de colaboração que ele mantém (DA SILVA, 2014). Uma ferramenta analítica utilizada para analisar os trabalhos publicados em coautoria é a teoria das redes sociais (SCOTT, 2012). A Análise de Redes Sociais (ARS) se baseia nas relações obtidas a partir dos atores que compõe a rede. Alguns aspectos podem ser identificados através da ARS, são eles: padrões de relacionamento e conectividade entre os atores de uma rede; formação de aglomerados; evolução da rede ao longo do tempo e, o fluxo de comunicação, informação e conhecimento dentro da rede. A ARS tem por objetivo modelar as conexões entre os atores para retratar, descrever e representar a estrutura do um grupo considerado (WASSERMAN; FAUST, 2007).

Um modelo de representação da organização das redes sociais pode ser obtido a partir da teoria dos grafos. Os grafos são bastante usados para representar redes sociais, pois permitem visualizar de forma simples as conexões entre os vértices da rede. Um grafo (G) pode ser definido como uma estrutura matemática composta por um conjunto não vazio de vértices ( $V$ ) que podem ou não estar ligados entre si por meio de arestas (A), ou seja, $G=(V, A)$ (GABARDO, 2015). Uma rede social de coautoria é formada quando pesquisadores publicam trabalhos científicos através coautoria. Neste tipo de rede, os vértices podem representar autores, instituições ou países. Adicionalmente, as arestas representam as conexões entre dois atores que fazem parte da rede. A ARS envolve três tarefas fundamentais: identificação de comunidades, detecção dos atores centrais e caracterização do papel de cada ator na rede (WATTENBERG, 2006).

Uma variável interessante a ser observada numa rede de coautoria é a componente gigante. Essa componente consiste no maior subgrafo conexo formado por um conjunto de vértices e arestas onde há, pelo menos, um caminho entre quaisquer pares de vértices. A componente gigante é o elemento com maior relevância na rede, pois todos os atores pertencentes a esse grupo estão conectados por atores em comum. Na rede de coautoria, essa medida é utilizada para investigar o comportamento dos autores participantes do maior grupo colaborativo da 
rede. Ela também permite verificar se a rede é coesa ou esparsa e dividida em pequenos grupos publicando isoladamente (NEWMAN, 2001).

\section{Metodologia}

As publicações sobre seis oleaginosas que fazem parte da cadeia de produção do biodiesel foram obtidas a partir do banco de dados Web of Science (WoS) do Institute for Scientific Information (ISI) Web of Knowledge. O WoS foi acessado através do portal de periódicos da CAPES. As oleaginosas selecionadas para este estudo foram: soja, pinhão-manso, dendê, canola, girassol e mamona. Todas as publicações compreendidas entre 1945 e 2011 foram investigadas. O levantamento foi iniciado em 1945, pois o WoS somente disponibiliza a produção científica a partir desse ano específico. Contudo, o intervalo de tempo estudado compreendeu 20 anos, devido a primeira publicação associando uma das olegionosas selecionadas ao biodiesel ter ocorrido somente no ano de 1992.

As consultas (nome popular ou científico da oleaginosa e biodiesel) foram feitas na Coleção Principal do WoS no modo "Pesquisa Avançada". O levantamento foi executado buscando-se termos específicos nos seguintes campos das publicações: TI (Título) and TS (Tópico). O campo TS realiza uma busca no Título, Resumo e/ou Palavras-chave da publicação. Assim, no título pesquisou-se o nome popular ou científico da oleaginosa relacionada e no campo tópico o vocábulo biodiesel. Logo, a pesquisa foi realizada da seguinte forma: para a soja o código utilizado foi [TI = (soybean or Glycine max) AND TS = (Biodiesel)], para o pinhão-manso $[\mathrm{TI}=$ (jatropha or Jatropha curcas) AND TS = (Biodiesel) $]$, para o dendê $[\mathrm{TI}=$ (palm or Elaeis guineensis) AND TS $=$ (Biodiesel) $]$, para o girassol $[\mathrm{TI}=$ (sunflower or Helianthus annuus) AND TS $=$ (Biodiesel) $]$, para a canola $[\mathrm{TI}=$ (rapeseed or Brassica napus) AND TS $=$ (Biodiesel) $]$, e para a mamona $[\mathrm{TI}=$ (castor or Ricinus communis) AND TS = (Biodiesel)].

Nesta pesquisa todos os tipos de documentos do ISI foram selecionados: artigos, revisões, editoriais, cartas, notas, dentre outros. Para cada publicação levantada, foram registradas o ano de publicação, nome e sobrenome dos autores e endereços incluindo as instituições e países de cada um. Esta informação foi organizada e inserida em uma planilha de dados, onde os seguintes indicadores bibliométricos foram computados por ano: quantidade de publicações, autores, países e instituições. O nome das instituições e países passaram por um processo de padronização detalhada para impossibilitar a geração de vértices múltiplos representando o mesmo ator. Esse processo de depuração tende a evitar problemas de imprecisão na geração da rede. As relações entre os vértices foram determinadas a partir da coautoria. Assim, caso haja dois autores em uma mesma publicação, os vértices que os representam serão ligados por uma aresta na rede dos autores.

Diversos programas computacionais têm sido desenvolvidos no intuito de facilitar o trabalho dos pesquisadores na construção, visualização e geração das redes de colaboração científica. Deste modo, o 
programa Network Workbench Tool (NWB, 2006) foi utilizado para gerar a rede de autores, pois ele é capaz de ler o formato do arquivo gerado pelo banco de dados WoS. Esta ferramenta pode ser acessada livremente durante o seu pré-processamento dos dados é realizado a remoção de registros duplicados. O NWB foi desenvolvido pela Universidade de Indiana localizada nos Estados Unidos da América (COBO et al., 2011). A rede de países e de instituições foi gerada através dos dados obtidos dos documentos investigados. Para a visualização e análise das três redes geradas os programas Gephi e Pajek foram utilizados. Estes dois programas são desenvolvidos em código aberto.

O Gephi é totalmente gratuito e foi desenvolvido em linguagem Java na interface de usuário do NetBeans. A primeira versão dessa ferramenta foi inicialmente desenvolvida por estudantes da Universidade de Tecnologia de Compiègne na França, no ano de 2008. Ele possui arquitetura flexível e multi-tarefa para trabalhar com conjunto de dados complexos. Ele foi desenvolvido em módulos que podem importar, visualizar, filtrar, manipular e exportar todo tipo de grafo (BASTIAN; HEYMANN; JACOMY, 2009). O Pajek pode ser utilizado gratuitamente para uso não-comercial e está em constante desenvolvimento desde 1996. Ele permite criar, editar e manipular grafos de tamanhos extremamente grandes. Essa ferramenta possui alguns algoritmos e heurísticas implementadas para auxiliar a descoberta de informações. 0 Pajek também possui grande quantidade de operações de ARS disponíveis para utilização (NOOY; MRVAR; BATAGELJ, 2011).

\section{Resultados e discussão}

Ao longo de todo o período estudado, esta pesquisa analisou 1378 publicações científicas. Esta consulta se iniciou em 1945, mas como a primeira publicação só ocorreu em 1992, ela analisou de fato o período compreendido entre 1992 e 2011, ou seja, 20 anos. Os resultados da Tabela 1 mostraram que houve um elevado crescimento das publicações registradas no banco de dados WoS sobre as seis oleaginosas pesquisadas que fazem parte da cadeia produtiva do biodiesel. O intervalo de tempo foi dividido em períodos de 4 anos, para uma melhor visualização dos dados. Até o ano de 1999, a canola foi a oleginosa associada a produção de biodiesel com maior quantidade de trabalhos científicos publicados.

A soja e o pinhão-manso representaram as oleaginosas com maior quantidade de trabalhos acadêmicos registrados, possuindo essas duas oleaginosas cerca de $50 \%$ do total de publicações. Ao se analisar os dois últimos períodos (2003 a 2007 em relação 2008 a 2011), observa-se que o pinhão-manso foi a oleaginosa com o maior acréscimo no número de publicações (aproximadamente $1283 \%$ ) e a soja obteve o menor aumento em relação a todas as oleaginosas estudadas (em torno de 194\%). Contudo, a soja se destaca como matéria-prima para produção de biodiesel no Brasil, pois ela corresponde aproximadamente $80 \%$ do volume total produzido deste biocombustível (ANP, 2014). 
Tabela 1 - Quantidade da publicação mundial por oleaginosa associada ao biodiesel

\begin{tabular}{c|c|c|c|c|c|c|c}
\hline Período & Soja & $\begin{array}{c}\text { Pinhão- } \\
\text { manso }\end{array}$ & Dendê & Canola & Girassol & Mamona & Total \\
\hline \hline $1992-1995$ & 1 & 0 & 0 & 2 & 0 & 0 & 3 \\
\hline $1996-1999$ & 5 & 2 & 0 & 15 & 4 & 0 & 26 \\
\hline $2000-2003$ & 14 & 1 & 6 & 16 & 8 & 0 & 45 \\
\hline $2004-2007$ & 85 & 23 & 26 & 21 & 20 & 16 & 191 \\
\hline $2008-2011$ & 250 & 318 & 245 & 99 & 120 & 81 & 1113 \\
\hline Total & 355 & 344 & 277 & 153 & 152 & 97 & 1378 \\
\hline
\end{tabular}

Fonte: Dados da pesquisa.

A Figura 1 apresenta os quatro países que mais publicaram sobre o tema pesquisado para cada oleaginosa. Para gerar este resultado, primeiramente todos os países por oleaginosa foram listados. Em seguida um somatório para contabilizar quantas ocorrências cada país possui foi realizado. Consequentemente, o total de vezes no qual cada país foi associado a uma publicação foi determinado. Os países em destaque na quantidade de trabalhos foram: Brasil, Índia, China e Estados Unidos da América. No intervalo de tempo analisado, os autores com endereço brasileiro foram os que mais publicaram sobre mamona associado a biodiesel, pois eles detiveram $56 \%$ da produção científica sobre este tema. Os autores com endereço indiano, chinês e norte americano foram os que mais publicaram sobre pinhão-manso, canola e soja, respectivamente.

Os 5 países que mais produziram biodiesel em 2014 foram nessa ordem: Estados Unidos da América, Brasil, Alemanha, Indonesia e Argentina. Logo, o Brasil que foi o segundo colocado em produção de biodiesel foi o primeiro em quantidade de publicações. Adicionalmente, 0 país que mais produziu esse biocombustível ficou em quarta colocação em número de trabalhos científicos. Os três países que mais publicaram associando as oleaginosas selecionadas ao biodiesel fazem parte do BRIC. Desse grupo de países emergentes ficou de fora apenas a Rússia. Isso mostra que estas nações estão incentivando pesquisas científicas sobre biodiesel.

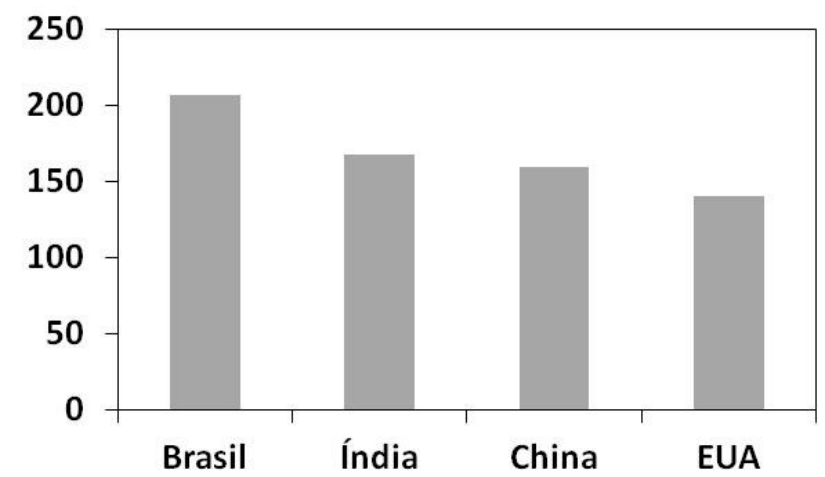

Figura 1 - Paises que mais publicaram associando as oleaginosas selecionadas ao biodiesel

Fonte: Dados da pesquisa. 


\subsection{Rede de Autores}

A Tabela 2 apresenta a distribuição de autores com publicações científicas sobre as oleaginosas selecionadas. Os resultados apresentaram produções variando de um autor até mais de 10 autores por documento analisado. Os dados mostraram também uma concentração de cooperação variando entre três e quatro autores, pois em torno de $47 \%$ de todos os trabalhos investigados foram publicados por essas duas quantidades de autores. Apenas aproximadamente 0,4 dos documentos examinados foram publicados por 1 autor, ou seja, sem compartilhamento de autoria. Os dados computados nesta tabela mostraram que as grandes colaborações científicas foram escassas neste campo do conhecimento.

Tabela 2 - Distribuição da quantidade de autores por oleaginosa

\begin{tabular}{c|c|c|c|c|c|c|c}
\hline $\begin{array}{c}\text { Autores por } \\
\text { publicação }\end{array}$ & Soja & $\begin{array}{c}\text { Pinhão- } \\
\text { Manso }\end{array}$ & Dendê & Canola & Girassol & Mamona & Total \\
\hline \hline $\mathbf{1}$ & 17 & 11 & 11 & 3 & 10 & 2 & 54 \\
\hline $\mathbf{2}$ & 48 & 58 & 54 & 33 & 17 & 14 & 224 \\
\hline $\mathbf{3}$ & 63 & 87 & 91 & 40 & 26 & 14 & 321 \\
\hline $\mathbf{4}$ & 91 & 80 & 67 & 29 & 35 & 18 & 320 \\
\hline $\mathbf{5}$ & 56 & 44 & 24 & 24 & 27 & 16 & 191 \\
\hline $\mathbf{6}$ & 35 & 27 & 16 & 10 & 15 & 16 & 119 \\
\hline $\mathbf{7}$ & 19 & 17 & 6 & 5 & 11 & 8 & 66 \\
\hline $\mathbf{8}$ & 14 & 12 & 4 & 4 & 6 & 6 & 46 \\
\hline $\mathbf{9}$ & 6 & 5 & 2 & 4 & 3 & 2 & 22 \\
\hline $\mathbf{1 0}$ ou mais & 6 & 3 & 2 & 1 & 2 & 1 & 15 \\
\hline Total & $\mathbf{3 5 5}$ & $\mathbf{3 4 4}$ & $\mathbf{2 7 7}$ & $\mathbf{1 5 3}$ & $\mathbf{1 5 2}$ & $\mathbf{9 7}$ & $\mathbf{1 3 7 8}$ \\
\hline
\end{tabular}

Fonte: Dados da pesquisa.

Os dados observados na rede de autores estão descritos na Tabela 3. Essa tabela apresenta a quantidade de autores distintos, comunidades e autores na maior comunidade por oleaginosa. $O$ pinhão-manso apresentou a maior comunidade, pois cerca de $8,7 \%$ do total de autores que publicaram sobre esta oleaginosa pertenciam a essa componente. A razão entre quantidade de comunidades e autores foi obtida e os resultados foram bem próximos para todas as oleaginosas (variando em torno de $19 \%$ ). A quantidade de autores publicando sobre pinhão-manso e soja equivale a cerca de $50 \%$ do total dos documentos levantados. O número de autores por publicação pode ser calculado pela razão entre a quantidade de autores distintos e a quantidade de publicações. Assim, com os dados das tabelas 1 e 3, o número médio de autores por publicação foi aproximadamente 2,9 para a soja; 3 para o pinhão manso; 2,4 para o dendê; 3,1 para a canola; 3,6 para o girassol e 3,6 para a mamona. 
Tabela 3 - Quantificação de autores e grupos de pesquisa por oleaginosa no período selecionado

\begin{tabular}{c|c|c|c|c}
\hline Oleaginosas & $\begin{array}{c}\text { Autores } \\
\text { Distintos }\end{array}$ & $\begin{array}{c}\text { \# de } \\
\text { Componentes }\end{array}$ & $\begin{array}{c}\text { \# de autores na maior } \\
\text { componente }\end{array}$ & $\begin{array}{c}\text { Razão entre } \\
\text { componentes e } \\
\text { autores }\end{array}$ \\
\hline \hline Soja & 1034 & 167 & 85 & $16,3 \%$ \\
\hline Pinhão-manso & 1038 & 184 & 90 & $17,8 \%$ \\
\hline Dendê & 664 & 134 & 31 & $20,1 \%$ \\
\hline Canola & 471 & 100 & 12 & $21,2 \%$ \\
\hline Girassol & 551 & 106 & 17 & $19,2 \%$ \\
\hline Mamona & 353 & 65 & 28 & $18,4 \%$ \\
\hline
\end{tabular}

Fonte: Dados da pesquisa.

\subsection{Rede de países}

A Tabela 4 exibe a distribuição da quantidade de países com publicações sobre cada oleaginosa pesquisada. No período analisado, essa pesquisa encontrou 1378 publicações. Contudo, somente um total de 1365 trabalhos possuia a identificação do país no campo endereço, pois não foi encontrado a informação sobre o país em 5 publicações sobre pinhão-manso, 4 sobre soja e 4 sobre dendê. Assim, 13 publicações ficou sem esta identificação e sem ser contabilizada nesta tabela. Os registros demonstraram que aproximadamente $88 \%$ do total de publicações verificadas foram realizadas por apenas um país. O pinhão-manso foi a única oleaginosa que possuiu colaboração entre mais de 3 países distintos. Este fato indicou que a colaboração internacional deve ser incentivada, pois ela possui pouca representatividade no universo investigado.

Tabela 4 - Distribuição da quantidade de países por oleaginosa

\begin{tabular}{c|c|c|c|c|c|c|c}
\hline & Soja & Pinhão-manso & Dendê & Canola & Girassol & Mamona & Total \\
\hline \hline $\mathbf{1}$ & 318 & 284 & 244 & 133 & 139 & 90 & 1208 \\
\hline $\mathbf{2}$ & 33 & 47 & 26 & 18 & 12 & 7 & 143 \\
\hline $\mathbf{3}$ & 0 & 3 & 3 & 2 & 1 & 0 & 9 \\
\hline $\mathbf{4}$ & 0 & 3 & 0 & 0 & 0 & 0 & 3 \\
\hline $\mathbf{5}$ & 0 & 2 & 0 & 0 & 0 & 0 & 2 \\
\hline Total & $\mathbf{3 5 1}$ & $\mathbf{3 3 9}$ & $\mathbf{2 7 3}$ & $\mathbf{1 5 3}$ & $\mathbf{1 5 2}$ & $\mathbf{9 7}$ & $\mathbf{1 3 6 5}$ \\
\hline
\end{tabular}

Fonte: Dados da pesquisa.

A Figura 2 apresenta as redes de países que realizaram publicações sobre as seis oleaginosas pesquisadas neste trabalho. Os quatro países (Brasil, Índia, China e EUA) que mais publicaram sobre o tema pesquisado (ver Figura 1) estão em destaque nas redes. Na rede da soja, a Índia não apresentou colaboração e o país no qual houve mais colaboração é os EUA, pois ele produziu em parceria com outras 9 nações. $O$ pinhão-manso apresentou o maior número de países distintos e o menor número de componentes. A componente gigante dessa oleaginosa abrangeu cerca de $76 \%$ do total de países. Nessa rede, a Índia cooperou com 13 países, a China com 9, os EUA com 3 e o Brasil publicou exclusivamente com a Nigéria. Na rede do dendê, a Malásia foi o país que mais colaborou. Adicionalmente, a China e a Índia não pertencem a componente principal. 
$\mathrm{Na}$ rede da canola, nenhum dos quatro países em destaque colaborou entre si e os autores com endereço indiano publicaram isoladamente. 0 girassol apresentou o maior número de componente e países sem cooperação internacional. Esse fato é comprovado, pois aproximadamente $65 \%$ dos países não pertencem a nenhuma componente. Na rede do girassol, o país mais conectado foi a Espanha com 6 ligações. A mamona possui a rede no qual todos os principais países colaboraram com algum outro.

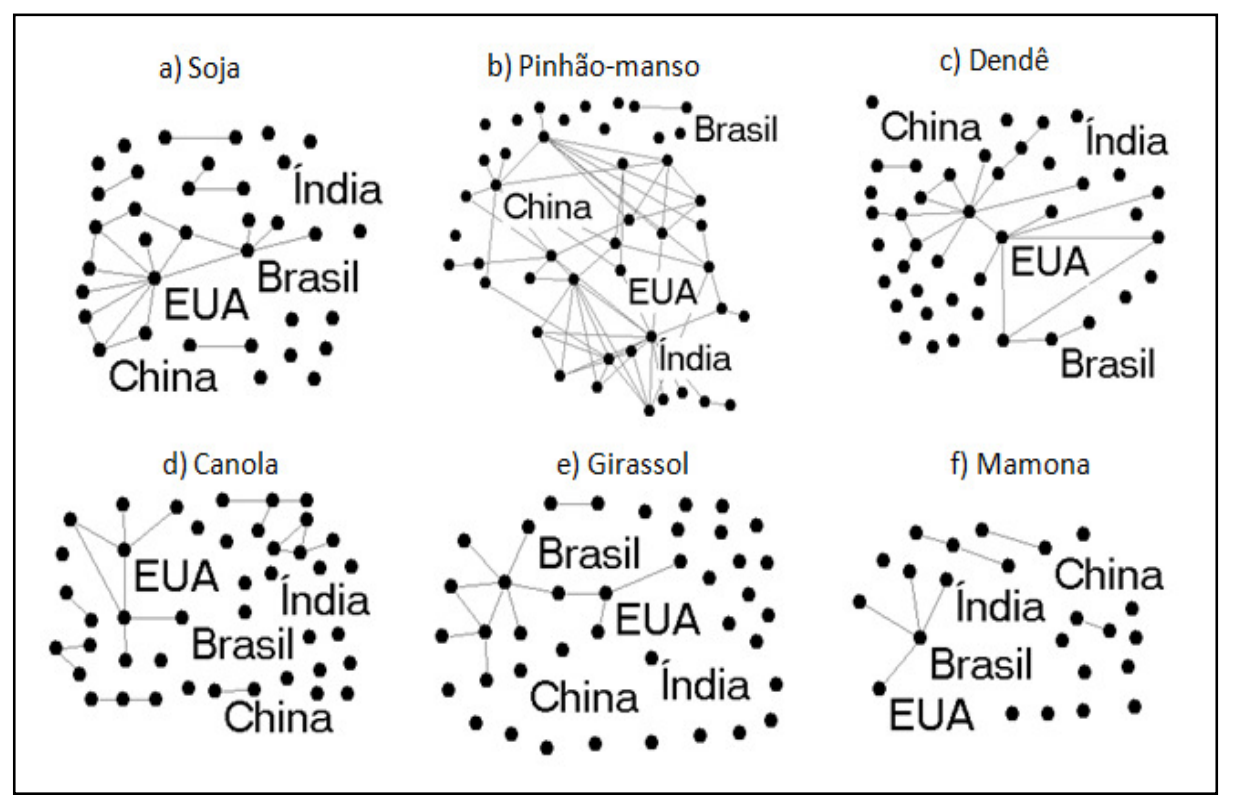

Figura 2 - Rede de países. a) Soja: 35 países, 17 componentes, principal com 14 países e 12 países sem colaboração. b) Pinhão-manso: 45 países, 11 componentes, principal com 34 países e 9 países sem colaboração. c) Dendê: 42 países, 21 componentes, principal com 21 países e 19 países sem colaboração. d) Canola: 42 países, 24 componentes, principal com 7 países e 17 países sem colaboração. e) Girassol: 40 países, 28 componentes, principal com 12 países e 26 países sem colaboração. e) Mamona: 23 países, 15 componentes, principal com 5 países e 11 países sem colaboração.

Fonte: Dados da pesquisa

\subsection{Rede de Instituições}

A Tabela 5 mostra a distribuição da quantidade de instituições por oleaginosa. Conforme já mencionado na Tabela 4, 13 publicações não possuíam a identificação da instituição e, assim, esses trabalhos não foram computados nesta tabela. As publicações registradas constataram que em torno de $60 \%$ dos trabalhos obtidos foram desenvolvidos por apenas uma instituição, ou seja, sem colaboração institucional. Os cerca de $40 \%$ dos trabalhos em colaboração foram assim distribuídos por quantidade de instituições participantes: por volta de $26 \%$ foram 
produzidos por 2 instituições, $10 \%$ por 3, 3\% por 4 e apenas $1 \%$ foram desenvolvidos por 5 ou mais instituições diferentes. Esse fato apresenta a necessidade de haver incentivo para as publicações interinstitucionais.

Tabela 5 - Distribuição da quantidade de instituições por oleaginosa

\begin{tabular}{c|c|c|c|c|c|c|c}
\hline & Soja & Pinhão-manso & Dendê & Canola & Girassol & Mamona & Total \\
\hline \hline $\mathbf{1}$ & 214 & 201 & 178 & 89 & 89 & 49 & $\mathbf{8 2 0}$ \\
\hline $\mathbf{2}$ & 83 & 91 & 68 & 43 & 41 & 26 & $\mathbf{3 5 2}$ \\
\hline $\mathbf{3}$ & 40 & 34 & 14 & 17 & 15 & 16 & $\mathbf{1 3 6}$ \\
\hline $\mathbf{4}$ & 12 & 10 & 7 & 4 & 6 & 6 & $\mathbf{4 5}$ \\
\hline $\mathbf{5}$ ou mais & 2 & 3 & 6 & 0 & 1 & 0 & $\mathbf{1 2}$ \\
\hline Total & $\mathbf{3 5 1}$ & $\mathbf{3 3 9}$ & $\mathbf{2 7 3}$ & $\mathbf{1 5 3}$ & $\mathbf{1 5 2}$ & $\mathbf{9 7}$ & $\mathbf{1 3 6 5}$ \\
\hline
\end{tabular}

Fonte: Dados da pesquisa.

As relações obtidas a partir da rede de instituições são apresentadas na Tabela 6. O pinhão-manso apresentou a maior quantidade de componentes e instituições entre as seis oleaginosas pesquisadas. Em torno de $37 \%$ das instituições que publicaram sobre dendê associado ao biodiesel não possuíram colaboração institucional, ou seja, o trabalho científico foi desenvolvido por um ou mais autores pertencentes ao mesmo estabelecimento. A canola e o girassol, apesar de ocupar a quarta e a quinta posição em relação ao número de instituições identificadas, possuíam somente 6 instituições na sua maior componente.

Tabela 6 - Relações obtidas a partir da rede de instituições

\begin{tabular}{c|c|c|c|c}
\hline Oleaginosas & $\begin{array}{c}\text { \# de } \\
\text { Instituições }\end{array}$ & $\begin{array}{c}\text { \# de } \\
\text { Componentes }\end{array}$ & $\begin{array}{c}\text { \# de Instituições na } \\
\text { maior Componentes }\end{array}$ & $\begin{array}{c}\text { Instituições sem } \\
\text { colaboração }\end{array}$ \\
\hline \hline Soja & 298 & 120 & 25 & 71 \\
\hline Pinhão-manso & 341 & 140 & 27 & 76 \\
\hline Dendê & 201 & 100 & 14 & 45 \\
\hline Canola & 178 & 87 & 6 & 47 \\
\hline Girassol & 186 & 90 & 6 & 21 \\
\hline Mamona & 116 & 50 & 11 & \\
\hline
\end{tabular}

Fonte: Dados da pesquisa.

Para a soja, pinhão-manso, dendê, canola, girassol e mamona as instituições que mais publicaram foram: Universidade Tsinghua da China com 14 documentos, Indian Institute of Technology da India com 25, Universiti Sains Malaysia com 40, University of Pardubice da Republica Checa com 12, Universidade de Belgrado na Sérvia com 7 e Universidade Federal da Paraíba com 8, respectivamente. O Brasil tem um destaque especial nas publicações sobre a mamona, pois as sete primeiras colocações foram ocupadas por instituições brasileiras.

\section{Considerações Finais}

O estudo apresentado mostrou o crescimento da produção acadêmica sobre biodiesel associado a seis oleaginosas (soja, pinhãomanso, dendê, canola, girassol e mamona), no período de 1945 a 2011 . O intervalo investigado abrangiu 20 anos, pois o primeiro trabalho foi 
publicado somente no ano de 1992. As publicações foram obtidas no banco de dados Web of Science do Institute for Scientific Information Web of Knowledge. Dessa forma, esta pesquisa analisou 1378 documentos, sendo a soja e o pinhão-manso as oleaginosas com maior quantidade de publicações. No primeiro período estudado (1992 a 1995) unicamente trabalhos sobre soja e canola foram recuperados. A maior parte deles foi publicada predominantemente por 3 ou 4 autores, 1 instituição e 1 país. A colaboração institucional e internacional ainda é reduzida, pois abrangeu somente em torno de $40 \%$ e $11,5 \%$ do total de documentos analisados, respectivamente. Brasil, Índia, China e Estados Unidos da América foram os quatro países com maior número de publicações. Este fato mostrou o interesse em pesquisas sobre biodiesel nos países em desenvolvimento.

\section{Agradecimentos}

Este trabalho teve suporte da Coordenação de Aperfeiçoamento de Pessoal de Nível Superior (CAPES) e da Fundação de Amparo à Pesquisa do Estado da Bahia (FAPESB).

\section{Referências}

ABBASI, A.; ALTMANN, J.; HWANG, J. Evaluating scholars based on their academic collaboration activities: two indices, the RC-index and the CC-index, for quantifying collaboration activities of researchers and scientific communities. Scientometrics, v. 83, p. 1-13, 2010.

AGÊNCIA NACIONAL DO PETRÓLEO, GÁS NATURAL E BIOCOMBUSTÍVEIS (ANP). Resolução ANP $\mathrm{n}^{\circ}$ 7. Biodiesel Standard. Diário Oficial da União, Brasília, 20 mar. 2008.

AGÊNCIA NACIONAL DO PETRÓlEO, GÁS NATURAL E BIOCOMBUSTÍVEIS (ANP). Anuário estatístico brasileiro do petróleo, gás natural e biocombustíveis: 2014. Rio de Janeiro: ANP, 2014.

ANDRADE, I. L.; GALVÃO, V. Rede de colaboração científica das principais oleaginosas envolvidas na produção do biodiesel no Brasil. Diálogos \& Ciência, v. 34, p. 24-27, 2014.

ARBOIT, A. E.; BUFREM, L. S. Produção de trabalhos científicos em eventos nacionais da área de ciência da informação. TransInformação, v. 23, n. 3, p. 207-217, 2011.

BALANCIERI, R. et al. A análise de redes de colaboração científica sob as novas tecnologias de informação e comunicação: um estudo na Plataforma Lattes. Ci. Inf., Brasília, v. 34, n. 1, p. 64-77, 2005.

BARBASTEFANO, R. G. et al. Impactos dos nomes nas propriedades de redes sociais: um estudo em rede de coautoria sobre sustentabilidade. Perspectivas em Ciência da Informação, v. 18, n. 3, p. 78-95, 2013. 
BASTIAN, M.; HEYMANN, S.; JACOMY, M. Gephi: an open source software for exploring and manipulating networks. ICWSM, v. 8, p. 361-362, 2009.

BEATRIZ, A.; ARAÚJO, Y. K.; de LIMA, D. P. Glicerol: um breve histórico e aplicação em sínteses estereosseletivas. Química Nova, v. 34, n. 2, p. 306-319, 2011.

BORDONS, M.; GOMEZ, I. Collaboration networked in science. In: CRONIN, B.; ATKINS, H. B. (Ed.). The web of knowledge: a festschrift in honor of Eugene Garfield. New Jersey: ASIS, 2000. p. 197-214.

BRAGA, P. F.; PEREIRA, H. B. B.; MORET, M. A. Difusão do conhecimento sob a perspectiva da teoria de redes: Mapeamento da produção científica a partir de uma base de periódicos da Física. Perspectivas em Gestão \& Conhecimento, v. 4, p. 148-160, 2014.

COBO, M. J. et al. Science mapping software tools: Review, analysis, and cooperative study among tools. Journal of the American Society for Information Science and Technology, v. 62, n. 7, p. 1382-1402, 2011.

DA SILVA, A. K. A. A dinâmica das redes sociais e as redes de coautoria. Perspectivas em Gestão \& Conhecimento, v. 4, p. 27-47, 2014.

DABDOUB, M. J.; BRONZEL, J. L.; RAMPIN, M. A. Biodiesel: visão crítica do status atual e perspectivas na academia e na indústria. Química Nova, v. 32, n. 3, p. 776-792, 2009.

DE SOUZA, L. S.; GALVÃO, V. Rede de colaboração científica na área de bioenergia no Brasil: um estudo baseado nos artigos do periódico Biomass \& Bioenergy. In: EXLER, R. B.; SAMPAIO, L. P.; TEIXEIRA, L. P. B. (Org.). Bioenergia: um diálogo renovável. Salvador: Vento Leste, 2014. v. 3. p. 90-99. FERRARI, R. A.; OLIVEIRA, V. S.; SCABIO, A. Biodiesel de soja: taxa de conversão em ésteres etílicos, caracterização físico-química e consumo em gerador de energia. Química Nova, v. 28, n. 1, p. 19-23, 2005.

GABARDO, A. C. Análise de redes sociais: uma visão computacional. São Paulo: Novatec, 2015. v. 1, p. 144.

GLANZEL, W. Coauthorship patterns and trends in the sciences (1980-1998): a bibliometric study with implications for database indexing and search strategies. Library trends, v. 50, n. 3, p. 461-475, 2002.

GOLDENBERG, J. et al. The evolving social network of marketing scholars. Marketing Science, v. 29, p. 561-567, 2010.

GONÇALVES, P. C. S. et al. Produção e uso dos diferentes tipos de biocombustíveis. In: EXLER, R. B.; SAMPAIO, L. P.; TEIXEIRA, L. P. B. (Org.). Bioenergia: um diálogo renovável. Salvador: Vento Leste, 2014. v. 3. p. 100-112. 
GONZÁLEZ-ALCAIDE, G. et al. Scientific authorships and collaboration network analysis on Chagas disease: papers indexed in pubmed (1940-2009). Revista do Instituto de Medicina. Tropical, v. 54, n. 4, p. 219-228, 2012.

GUIMARÃES, C. M. M.; GALVÃO, V. Colaboração científica sobre biogás no Brasil. In: EXLER, R. B.; SAMPAIO, L. P.; TEIXEIRA, L. P. B. (Org.). Bioenergia: um diálogo renovável. Salvador: Vento Leste, v. 3. p. 128-139, 2014.

GUIMARÃES, C. M.; GALVÃO, V. Análise da rede de colaboração científica sobre biogás. Perspectivas em Ciência da Informação, v. 20, n. 2, p. 120-133, 2015.

HAYASHI, M. A. P. I.; HAYASHI, C. R. M.; LIMA, M. Y. Análise de redes de co-autoria na produção científica em educação Especial. Liinc em Revista, v. 4, n. 1, p. 84-103, 2008.

HOLANDA, A. Biodiesel e inclusão social. Brasília: Câmara dos Deputados, 2004. p. 202. (Série Cadernos de Altos Estudos, n. $1)$.

JOHNSON, J. D. Análise de redes. In: JOHNSON, J. D. (Org.). Gestão de redes de conhecimento. São Paulo: Senac, 2011. p. 47-80.

KATZ, J. S.; MARTIN, B. R. What is research collaboration? Research Policy, Amsterdam, v. 26, p. 1-18, 1997.

LAUDEL, G. What do we measure by co-authorships? Research Evaluation, v. 11, n. 1, p. 3-15, 2002.

LETA, J.; CHAIMOVICH, $H$. Recognition and international collaboration: the Brazilian case. Scientometrics, v. 53, n. 3, p. 325-335, 2002.

LOBO, I. P.; FERREIRA, S. L. C.; CRUZ, R. S. Biodiesel: parâmetros de qualidade e métodos analíticos. Química Nova, v. 32, p. 1596-1608, 2009.

MAIA, M. F. S.; CAREGNATO, S. E. Co-autoria como indicador de redes de colaboração científica. Perspectivas em Ciência da Informação, v. 13, n. 2, p. 18-31, 2008.

MARCELO, J. F., HAYASHI, M. C. P. I. Estudo bibliométrico sobre a produção Científica no campo da sociologia da Ciência. Informação \& Informação, v. 18, n. 3, p. 138-153, 2013.

MOREL, C. M. et al. Co-authorship network analysis: a powerful tool for strategic planning of research, development and capacity building programs on neglecteddiseases. PLoS Neglected Tropical Diseases, v. 3, n. 8, e501, 2009.

NETWORK WORKBENCH TOOL (NWB). Versão 1.0.0. Michigan: Indiana University, Northeastern University; University of Michigan, 2006. Disponível em: <http://nwb.cns.iu.edu/>. Acesso em: 15 jun. 2015.

NEWMAN, M. E. The structure of scientic collaboration networks. Proceedings of the National Academy of Sciences of the United States of America, v. 98, n. 2, p. 404-409, 2001. 
NOOY, W.; MRVAR, A.; BATAGELJ, V. Exploratory social network analysis with Pajek. England; New York: Cambridge University Press, 2011.

OTTE, E.; ROUSSEAU, R. Social network analysis: a powerful strategy, also for the information sciences. Journal of Information Science, Cambridge, v. 28, n. 6, p. 441-453, 2002.

PERLINGEIRO, C. A. G. Biocombustíveis no Brasil: fundamentos, aplicações e perspectiva. Rio de Janeiro: Synergia, 2014.

PERSSON, O.; GLÄNZEL, W.; DANELL, R. Inflationary bibliometric values: the role of scientific collaboration and the need for relative indicators in evaluative studies. Scientometrics, v. 60, n. 3, p. 421-432, 2004.

POUSA, G. P. A. G.; SANTOS, A. L. F.; SUAREZ, P. A. Z. History and policy of biodiesel in Brazil. Energy Policy, v. 35, p. 5393-5398, 2007.

PUPPAN, D. Environmental evaluation of biofuels. Periodica Polytechnical Social and Management Sciences, v. 10, p. 95116, 2002.

RAMOS, J. M.; GONZÁLEZ-ALCAIDE, G.; BOLAÑOS-PIZARRO, $M$. Bibliometric analysis of leishmaniasis research in Medline (1945-2010). Parasites \& Vectors, v. 6, p. 55, 2013.

REVERTER-MASÍA, J. et al. Producción de artículos en la base de datos Web of Science y Scopus sobre educación física: estudio comparativo entre España y Brasil. TransInformação, v. 26, n. 2, p. 113-124, 2014.

SAlES, C. A. C.; AMORIM, M. C.; PINTO, C. H. C. Produção científica ligada ao biodiesel: classificação de artigos publicados em bases de dados internacionais entre os anos de 1998 e 2006. In: SIMPÓSIO DE ENGENHARIA DE PRODUÇÃO (SIMPEP), 13., Bauru, 2006. Anais... Bauru, 2006.

SANTIN, D. M.; VANZ, S. A. de S.; STUMPF, I. R. C. Produção científica em Ciências Biológicas da UFRGS: tendências temáticas no período 2000-2011. Perspectivas em Ciência da Informação, v. 20, n. 3, p. 3-21, 2015.

SCOTT, J. Social network analysis. 3. ed. Reino Unido: Sage Publications, 2012.

SILVA. A. O. et al. Colaboração entre programas de pósgraduação brasileiros em ciência da informação: modelagem baseada em grafos. Informação \& Informação, Londrina, v. 17, n. 3, p. 1-22, 2012.

SILVA, Z. O. F.; GALVÃO, V. Biodiesel: uma análise sobre os principais países produtores. In: EXLER, R. B.; SAMPAIO, L. P. ; TEIXEIRA, L. P. B. (Org.). Bioenergia: um diálogo renovável. Salvador: Vento Leste, 2014. v. 3, p. 90-99.

SOUZA, C. G.; BARBASTEFANO, R. G.; LIMA, L. S. Knowledge diffusion and collaboration networks on life cycle assessment. 
The International Journal of Life Cycle Assessment, v. 16, n. 6, p. 561-568, 2011.

SOUZA, C. G.; BARBASTEFANO, R. G.; LIMA, L. S. Redes de colaboração científica na área de química no Brasil: um estudo baseado nas coautorias dos artigos da revista Química Nova. Química Nova, v.35, n.4, p.671-676, 2012.

TÁVORA, F. L. História e economia dos biocombustíveis no Brasil. Brasília: Centros de Estudos e Consultoria do Senado, 2011.

VANZ, S. A. S.; STUMPF, I. R. C. Colaboração científica: revisão teórico conceitual. Perspectivas em Ciência da Informação, v. 15, n. 2, p. 42-55, 2010.

VASCONCELLOS, A. G.; MOREL, C. M. Enabling policy planning and innovation management through patent information and co-authorship network analyses: a study of tuberculosis in Brazil. PLoS ONE, v. 7, n. 10, e45569, 2012.

WASSERMAN, S.; FAUST, K. Social network analysis: methods and applications. 16th ed. New York: Cambridge University Press, 2007.

WATTENBERG, M. Visual exploration of multivariate graphs. Proc. of SIGCHI Conf. Human Factors in Computing Systems, New York, ACM, p. 811-819, 2006.

WOSZEZENKI, C.; GONÇALVES, A. L. Mineração de textos biomédicos: uma revisão bibliométrica. Perspectivas em Ciência da Informação, v. 18, p. 24-44, 2013. 